\title{
Examining the Smoking and Vaping Behaviors and Preferences of Vape Shop Customers
}

\author{
Theodore Lee Wogener ${ }^{1}$, Roees A Shaikh ${ }^{2}$, Ellen Meier ${ }^{3}$, Alayna P Tackett ${ }^{1,4}$, Noor N Tahirkheli ${ }^{1}$, Eleanor L. \\ Leavens $^{2,4}$, Leslie Driskill ${ }^{1}$
}

\begin{abstract}
INTRODUCTION The prevalence of vape shops has increased dramatically in recent years. Yet, few studies have examined the smoking/vaping behaviors and vaping preferences of vape shop customers and the reasons they patronize vape shops. The present study seeks to address this gap.

METHODS A convenience sample of 100 vape shop customers completed a brief questionnaire assessing past and current tobacco/e-cigarette use, vaping preferences and reasons they patronize vape shops. Self-reported smoking status was confirmed via exhaled carbon monoxide measurement.

RESULTS Participants reported health and the need to quit/reduce smoking as the most important reasons for initiating e-cigarette use. Approximately $62 \%$ were biochemically-verified smoking abstinent and 100\% were using newer generation e-cigarette devices. A majority (91\%) reported enjoying vaping more than smoking, $80 \%$ preferred non-tobacco e-cigarette flavors, though $60 \%$ reported they would still vape if the only flavor available was tobacco. Participants reported "battery life", "tastes good", and "curbs cravings" as the most important e-cigarette features; the least important feature was "feels/looks like traditional cigarette". A majority reported they preferred to buy e-cigarette supplies at vapor shops because of "access to staff that can help troubleshoot e-cigarette problems" (85\%), and "enjoy the atmosphere" (74\%).

conclusions Vape shop customers demonstrated a high rate of smoking abstinence and reported product preferences consistent with newer generation devices. Customers valued the atmosphere and the knowledgeable staff at vape shops. These findings suggest important avenues of investigation into the effects of unique characteristics of vape shops on smoking and vaping behaviors of their patrons, not only for clinical research but also for tobacco regulation.
\end{abstract}

\author{
AFFILIATION \\ 1 University of Oklahoma \\ Health Sciences Center, \\ Department of Pediatrics, \\ Oklahoma Tobacco Research \\ Center, Oklahoma, USA \\ 2 University of Oklahoma \\ Health Sciences Center, \\ Oklahoma Tobacco Research \\ Center, Oklahoma, USA \\ 3 University of Minnesota, \\ Department of Psychiatry, \\ Minneapolis, Minnesota, USA \\ 4 Oklahoma State University, \\ Department of Psychology, \\ Stillwater, Oklahoma, USA

\section{CORRESPONDENCE TO \\ Dr. Theodore Lee Wagener, University of Oklahoma Health Sciences Center, Department of Pediatrics, Oklahoma Tobacco Research Center, 655 Research Parkway, Suite 400, 73104 Oklahoma City, United States. Email: theodore-wagener@ ouhsc.edu}

\section{KEYWORDS}

Smoking cessation, nicotine, vape shop, electronic cigarette, Electronic Nicotine Delivery Systems (ENDS)

\section{INTRODUCTION}

The prevalence of electronic cigarette (e-cigarette) use in the Unites States continues to rise among both youth and adults ${ }^{1}$ ${ }^{2}$. Over half of U.S. smokers have tried an e-cigarette and approximately $20 \%$ "vaped" in the past 30 days $^{3}$. At the same time, there has been a surge in the number of stores dedicated exclusively to the sale of e-cigarette products (i.e., vape shops), and it is estimated that over 10,000 U.S. vape shops have opened during the past decade, representing an estimated onethird of the e-cigarette market and its fastest growing sector ${ }^{4,5}$.
Vape shops differ from other sources of e-cigarette sales, like convenience stores or online markets. Unlike convenience stores, vape shops provide a wide variety of flavors, typically only sell newer generation devices (e.g., tank systems, mechanical mods, advanced personal vaporizers), and most do not sell e-cigarettes manufactured by the tobacco industry ${ }^{6,9}$. Unlike online retailers, vape shops offer a more interactive experience for their customers such as personal interaction with staff members who often also vape, sampling of e-liquids, and customization of vapor devices ${ }^{9-11}$. Vape 
shops also allow vaping inside the store and many provide customers the opportunity to socialize and participate in vape-related events such as cloud chasing competitions ${ }^{6}$. Lastly, vape shops often promote their products at point-ofsale as a way to quit smoking, with staff providing their own testimonials of how they were successful in quitting smoking with the use of e-cigarettes ${ }^{6,12,13}$. Each of these unique features of vape shops may influence the attitudes, beliefs and tobacco use behaviors of vape shop customers.

There is also concern that vape shops may have negative implications on public health. A recent systematic review and meta-analysis suggested that e-cigarette use is often associated with dual use and a lower likelihood of quitting smoking ${ }^{14}$. If the use of e-cigarettes leads to prolonging the use of combustible cigarettes, either through deferred cessation or dual use, or by enticing non-tobacco users to begin the use of nicotine and eventually begin smoking, then vape shops may indirectly increase the public health harm caused by combustible cigarettes. As a result, research is needed to characterize the tobacco use behaviors of vape shop customers.

To date, the research base examining vape shops has primarily examined vape shop retailers' tobacco-related attitudes and beliefs and marketing practices. Only recently have studies begun to examine the characteristics of the vape shop customer ${ }^{15,16}$. To begin to fullfill this research need, the present study examined vape shop customers' smoking and vaping behaviors, their vaping preferences, and reasons for patronizing vape shops.

\section{METHODS}

\section{Participants and Procedure}

Letters of support for data collection were obtained from four vapor shops located in a Southern Plains state. Using a convenience sample approach, all customers entering the vape shop were invited to participate by a study research assistant. Of those approached, $24 \%$ refused participation in the study, with "too busy" and "in a rush" being the most frequently reported reasons for not participating. All study data were collected in the fall of 2013 and spring of 2014. Recruitment was conducted on weekdays in 2-3 hour increments between $11 \mathrm{am}-2 \mathrm{pm}$. These hours were selected per consultation with vape shop owners who reported this as the time of day with the highest patronage. Participants were asked to complete both a paper and pencil survey and exhaled carbon monoxide (eCO) testing. Data collection was done outside of the store and participants were compensated $\$ 5$ for participating in the study. All study procedures were approved by the University's Institutional Review Board.

\section{Measures}

\section{Vaping Questionnaire}

Participants completed a modified questionnaire used in a previous study of vape shop patrons ${ }^{16}$. The questionnaire assessed demographic characteristics, including age, ethnicity, income, employment status and education level. Current smoking and vaping use behaviors and history were also assessed using self-reported typical number of cigarettes smoked per day before initiating e-cigarette use, typical number of cigarette smoked per day currently, estimated milliliters of e-liquid used per day, and initial and current e-liquid flavor (tobacco vs. non-tobacco). Current cigarette and e-cigarette dependence was measured with two questions assessing the time from waking until first daily cigarette (the Fagerström Test for Nicotine Dependence time to first cigarette, FTND-TTFC ${ }^{17,18}$ ) or e-cigarette (e-cigarette modified FTNDTTFC). Participants reported their duration of e-cigarette use ("How long have you been vaping?" open text categorized as years, months, and days), the first e-liquid tried (i.e., "When did you first started vaping, which flavor did you try first?"), interest in continuing to vape if tobacco was the only available e-liquid flavor, and preferences for using combustible versus e-cigarette device and why (i.e, "Do you enjoy vaping more than cigarettes? If yes, then what is the most important reason why?"). Additional information regarding location (i.e., vape shop, online, convenience store/grocery store, gas station, mall kiosk, other) of first e-cigarette purchased ("Where did you purchase your first e-cigarette/vaporizer product?"); rating on a scale from 1 ("not at all important") to 10 ("very important) desired features of an e-cigarette device (battery life, high voltage battery, looks cool, large in size, large tank capacity, curbs cravings, feels/looks like a traditional cigarette, has many interchangeable parts, lightweight, easy to use, small in size, gives a bigger hit, durable parts, tastes good); and primary reason for initiating e-cigarette use was collected (open text categorized as stop/reduce smoking, improve health, social stigma/peer pressure to quit smoking, save money, better taste, other). Lastly, using a Likert scale ranging from "strongly agree" to "strongly disagree" participants were asked to rate reasons for purchasing e-cigarette products from a vape shop (e.g., "I prefer to buy my e-cigarette/vaporizer products at a vape shop because the cost is lower"; "I prefer to buy at a vape shop because I can order a customizable nicotine level").

\section{Biochemical verification of self-reported smoking status} Following the recommendations of the Society for Research on Nicotine and Tobacco (SRNT) Subcommittee on Biochemical Verification of tobacco use and cessation ${ }^{19}$, especially when 
examining new products and products purported to reduce harm, self-reported abstinence was verified with eCO. The half-life of $\mathrm{CO}$ is up to 8 hours depending on a variety of factors (e.g., time of day, daily smoking rate, recency of smoking, physical activity), and studies have shown that expired $\mathrm{CO}$ is a valid indicator of smoking status and cessation outcomes and compares favorably with cotinine and other biochemical measures that have longer detection windows ${ }^{19,20}$. eCO was collected using a BedfontpiCO+ Smokerlyzer, andeCO $<$ $10 \mathrm{ppm}$ was the cut-off used to confirm self-reported smoking abstinence ${ }^{19,20}$. Participants who refused eCO verification $(n=6)$, were excluded from analyses examining self-reported quit status.

\section{Statistical Analyses}

Analyses were conducted using SPSS version 20.0 (SPSS Ins, Chicago, IL). Descriptive analyses were conducted for participants' demographic information, vaping/smoking history, smoking status, and vaping preferences. A one-way between-groups analysis of variance (ANOVA) with Tukey's post hoc comparisons was conducted to examine self-reported importance of select e-cigarette product features.

\section{RESULTS}

\section{Participant Characteristics}

One hundred vape shop customers (51\% male; 76\% White) completed the survey. The average participant age was 37.6 years $(\mathrm{SD}=15.1), 71 \%$ had completed at least some college, $78 \%$ were currently employed, and $65 \%$ were making $\geq$ $\$ 30,000$ a year. See Table 1 for complete demographic information.

\section{Vaping and Smoking History}

Participants had been vaping for an average of 14.6 months ( $\mathrm{SD}=9.7$ months; range $=1$ day -3.5 years $)$ and reported consuming an average of $8.1 \mathrm{~mL}(\mathrm{SD}=12.4)$ of e-liquid per day. All but three participants were cigarette smokers at the time they initiated e-cigarette use (Mcig/day $=19.4$, $\mathrm{SD}=12.6$ ); of the three who were not cigarette smokers at the time of e-cigarette initiation, one was a never tobacco user, one was a cigar smoker, and the other was a snus user. Among participants who were smokers at the time of initiating e-cigarette use, TTFC before they started vaping indicated moderate dependence, $\mathrm{M}=1.88$ ( $\mathrm{SD}=0.94)$. All participants were currently using a tank-style e-cigarette,

\section{Figure 1. Importance of Select e-cigarette Features}

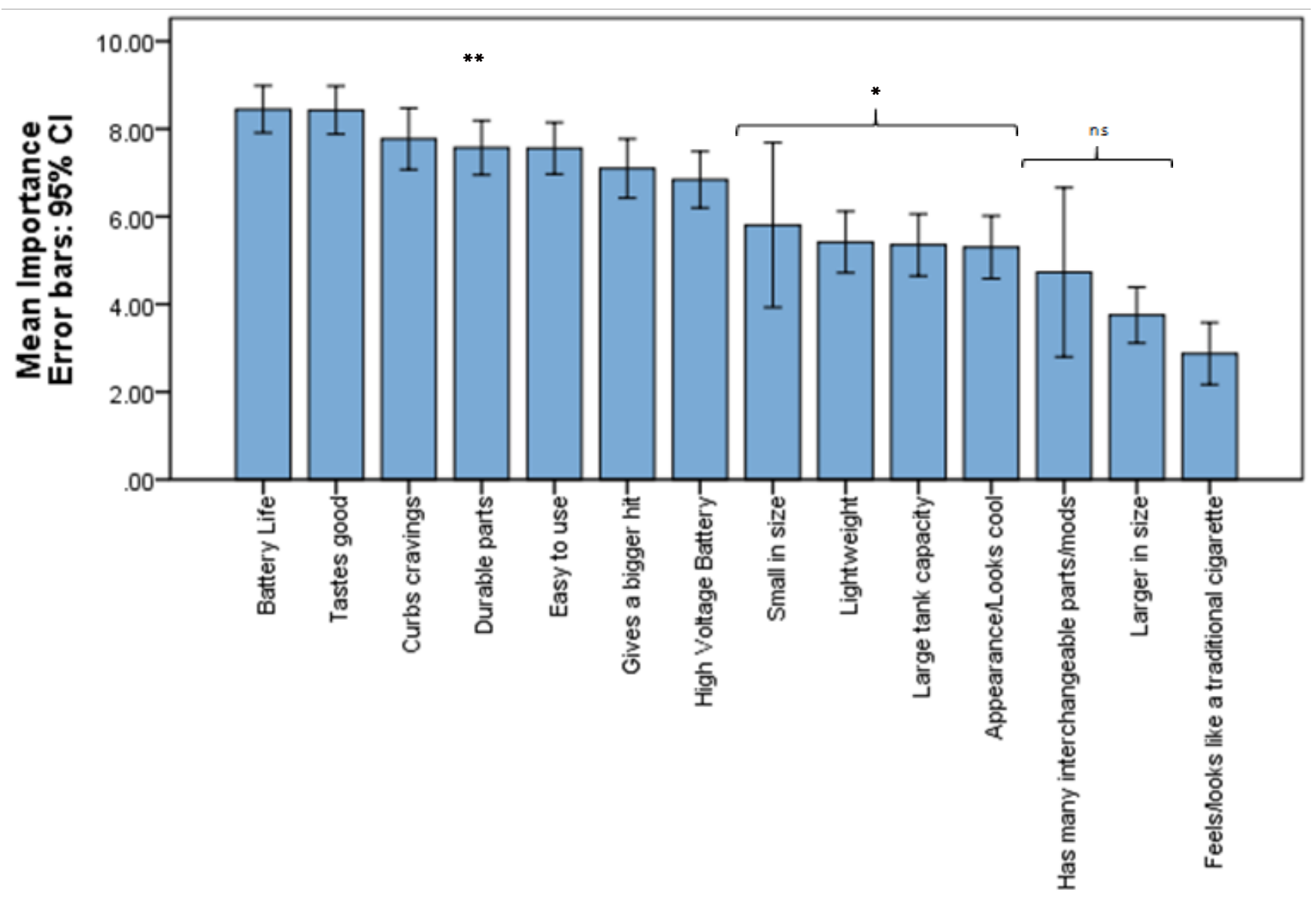

Note: All feature comparisons are made against "Feels/looks like a traditional cigarette"

${ }^{* *} p<.0001,{ }^{*} p<.05$, ns $=$ not significant $(p>.05)$. 


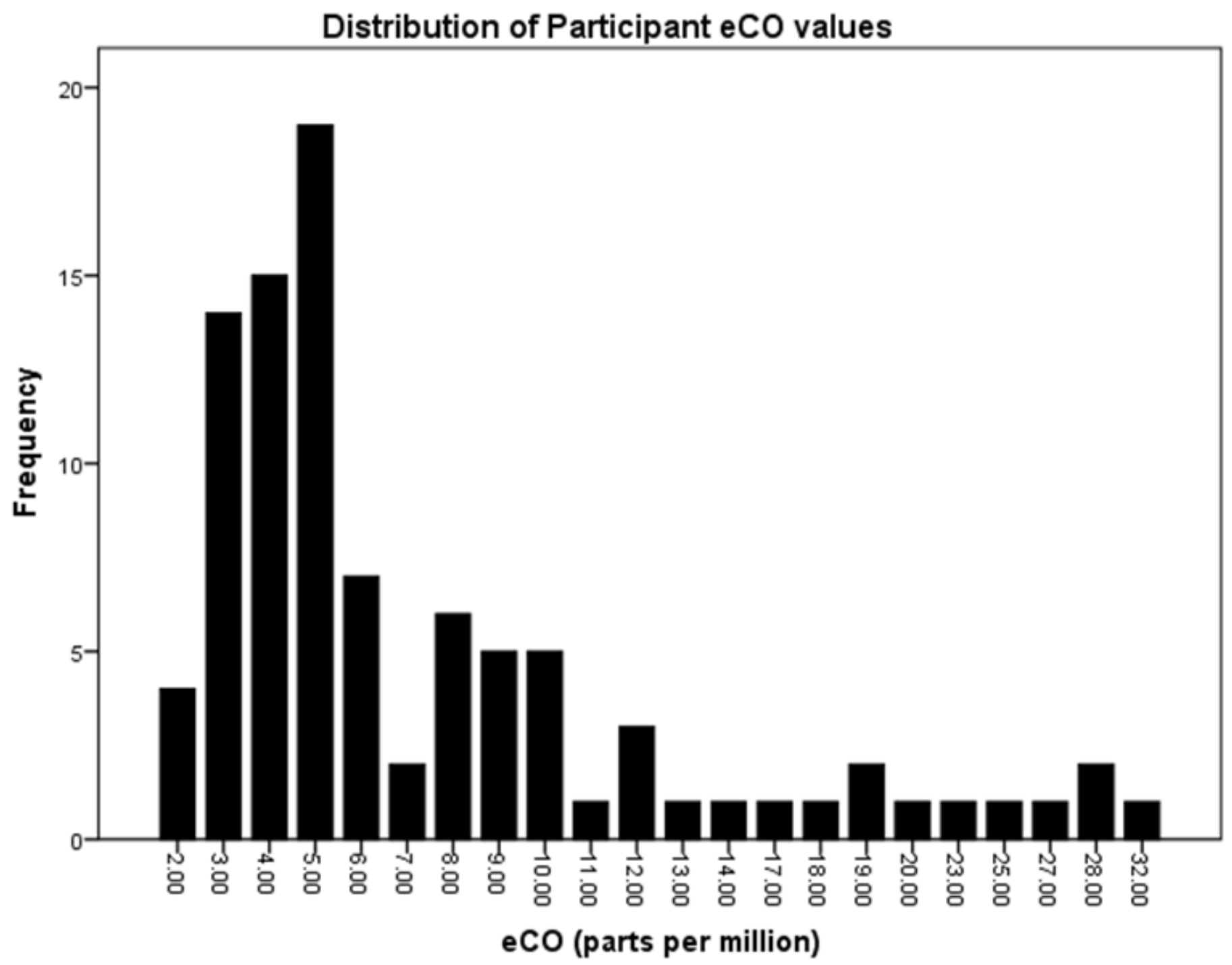

but $26 \%$ initiated use of e-cigarettes with a "cig-a-like" style product. Seventy-one percent purchased their first e-cigarette at a vape shop, while $11 \%$ purchased it at a convenience/ grocery store, $9 \%$ purchased online, $2 \%$ gas station, $2 \%$ smoke shop, and 5\% other (e.g., tattoo parlor).

\section{Reasons for Vaping}

Most participants reported that they started vaping to stop/ reduce smoking (48\%) and to improve their health (32\%). Additional reasons included social stigma/peer pressure to quit smoking $(10 \%)$, to save money $(4 \%)$, better taste (3\%), other (3\%, convenience, recreational use, avoid cleanindoor air laws).

\section{Smoking Status}

The majority of participants $(n=98)$ reported previous combustible tobacco use (e.g., cigarettes, cigars, hookah). One hundred participants were asked to complete eCO measurement, with six refusals. Of those who were using combustible tobacco before initiating e-cigarette use and completed eCO $(n=91), 65.9 \% \quad(n=60)$ selfreported smoking abstinence and eCO readings confirmed that $62.2 \% \quad(n=56)$ were not smoking (Mco=4.92ppm, $\mathrm{SD}=2.0)$. Among those who continued to smoke, mean cigarettes per day decreased from 19.0 to $4.8(\mathrm{p}<0.0001)$ since using e-cigarettes and mean eCO levels were 13.0 ppm ( $\mathrm{SD}=8 \cdot 2)$. See Figure 2 for the sample's distribution of eCO values.

\section{Vaping Preferences \& Vaping-related Social Activities} Forty percent of participants reported using tobacco flavored e-liquid when they first started vaping and 60\% reported that they would still vape if the only e-liquid flavor available was tobacco; however, $80 \%$ currently use and prefer a nontobacco flavored e-liquid. The majority of customers (91\%) reported enjoying vaping more than smoking. 
Table 1. Participant Demographics and Vaping/Smoking History $(n=100)$

\begin{tabular}{|c|c|}
\hline Variables & $\%$ or mean (SD) \\
\hline Age (years) & $37.6(15.1)$ \\
\hline Sex $(\%$ male $)$ & $51 \%$ \\
\hline \multicolumn{2}{|l|}{ Ethnicity } \\
\hline Caucosian & $76.0 \%$ \\
\hline African American & $10.0 \%$ \\
\hline Hispanic & $2.0 \%$ \\
\hline Asian & $2.0 \%$ \\
\hline American Indian/Alaskan Native & $5.0 \%$ \\
\hline Other & $5.0 \%$ \\
\hline \multicolumn{2}{|c|}{ Level of Education } \\
\hline Less than high school & $5.0 \%$ \\
\hline High school diploma/GED & $24.0 \%$ \\
\hline Some College & $28.0 \%$ \\
\hline College graduate & $23.0 \%$ \\
\hline Graduate College & $10.0 \%$ \\
\hline Technical/business school & $10.0 \%$ \\
\hline \multicolumn{2}{|c|}{ Household Income } \\
\hline$\$ 0-29999$ & $34.7 \%$ \\
\hline$\$ 30000-59999$ & $30.5 \%$ \\
\hline$\$ 60000-79999$ & $13.7 \%$ \\
\hline$\$ 80000$ or more & $21.1 \%$ \\
\hline \multicolumn{2}{|c|}{ Employment Status } \\
\hline Employed & $78.0 \%$ \\
\hline Unemployed & $6.0 \%$ \\
\hline Other (homemaker, retired, student) & $16.0 \%$ \\
\hline Vaping duration (months) & $14.6(9.71)$ \\
\hline \multicolumn{2}{|c|}{ Cigarettes per day } \\
\hline Before vaping & $19.4(12.6)$ \\
\hline After vaping & $4.8(3.6)$ \\
\hline \multicolumn{2}{|c|}{ Time to First Cigarette } \\
\hline Before vaping & $1.87(0.94)$ \\
\hline After vaping & $2.87(1.26)$ \\
\hline \multicolumn{2}{|c|}{ Source of first e-cigarette/vaporizer purchase } \\
\hline Vape shop & $70.7 \%$ \\
\hline Online & $9.1 \%$ \\
\hline Convenience/grocery store & $10.1 \%$ \\
\hline Gas station & $2.0 \%$ \\
\hline Other (smoke shop, tattoo parlor etc) & $8.1 \%$ \\
\hline \multicolumn{2}{|c|}{ First vape flavor tried } \\
\hline Tobacco & $40.4 \%$ \\
\hline Other than tobacco & $59.6 \%$ \\
\hline
\end{tabular}

GED = general education development; SD = standard deviation .
Participants reported that the most important e-cigarette features were "battery life" $(\mathrm{M}=8.5, \mathrm{SD}=2.6)$, "tastes good" ( $\mathrm{M}=8.4, \mathrm{SD}=2.6)$, and "curbs cravings" $(\mathrm{M}=7.8, \mathrm{SD}=3.3)$; the least important feature was "feels/looks like traditional cigarette" ( $\mathrm{M}=2.9, \mathrm{SD}=3.2)$. Battery life, having a high voltage battery, larger tank capacity, the ability to curb cravings, made of durable parts, and provides a bigger throat hit were all significantly more important than looking or feeling like a traditional cigarette $(\mathrm{p} \leq 0.01)$. See Figure 1 for mean importance values of select e-cigarette features.

A majority of participants 'agreed' or 'strongly agreed' that they preferred to buy e-cigarette supplies at vape shops because "the staff can help me troubleshoot my e-cigarette if I have problems" (85\%), "they are knowledge able about vaping and also vape" (74\%), "enjoy the atmosphere there" (70\%), "the e-liquid is fresher" (66\%), "I can order a customizable nicotine level" (54\%), "the e-liquid is prepared in-house" (50\%). Lower cost (20\%) and "more flavors available" (17\%) were much less important.

\section{DISCUSSION}

Vape shops are the fastest growing sector of the e-cigarette marketplace and the present study is one of the first to examine the characteristics of vape shop customers including their vaping behaviors and preferences. The reasons provided by customers for preferring to buy their products from vape shops such as the environment, advice, and support from vape shop staff, were consistent with the unique characteristics that distinguish vape shops from convenience stores and online stores $^{9,13}$.

While the cross-sectional, uncontrolled nature of this study precludes any causal link between e-cigarette use and quitting, there was a high rate of self-reported smoking abstinence (66\%) among this sample of vapers who reported smoking at the time of e-cigarette initiation. Following the recommendations of the SRNT Subcommittee on Biochemical Verification ${ }^{19}$, self-reported abstinence was verified with eCO and confirmed smoking abstinence among $62 \%$ of the sample. This finding should be interpreted with caution as there is a strong likelihood of self-selection bias, with customers who like and have found e-cigarettes helpful returning to vape shops while those who found them to be unhelpful not returning. Moreover, a recent meta-analysis and national survey showed reduced likelihood of quitting smoking among those who tried e-cigarettes ${ }^{14}$ as well as low product uptake ${ }^{21}$. Nonetheless, this finding points to the need for further prospective and controlled research on the smoking behaviors of vape shop customers, especially examining how the unique features 
of vape shops, such as the personal interaction between customers and sales personnel who are knowledgeable about and who have a highly favorable opinion of e-cigarettes ${ }^{10,11}$, 22, and vape shop products (advanced e-cigarette devices with improved nicotine delivery) influence customers' vaping and smoking patterns. To date, only one uncontrolled, prospective vape shop study has been conducted but demonstrated a $41 \%$ intention-to-treat quit rate of combustible cigarettes following 12 -months of vaping ${ }^{23}$.

Participants' e-cigarette product feature preferences, such as "long battery life", "curbs cravings", and "gives a bigger hit" were not surprising and were consistent with features one would assume newer generation device users would prefer. Interestingly, while users also rated "tastes good" as the second most important product feature, $60 \%$ of participants said they would still vape if the only available e-liquid flavor was tobacco-flavor. It is not immediately clear why this is the case, as participants were not questioned further about their reasons in the survey. However, it may be that while taste is an important product feature, ultimately, and as observed in the present sample, participants are vaping to improve their health and to quit smoking, not just as a recreational activity. If this finding is confirmed by future research, it may have implications for future US FDA regulations by suggesting that a potential flavoring ban on e-cigarette products may not lead e-cigarette users to abandon vapor devices and return to smoking as some suggest ${ }^{8,24}$. It is also worth noting that $60 \%$ of our participants reported using a non-tobacco flavor when they first started vaping. This finding further reiterates the importance of future research examining the potential of nontobacco flavorings attracting new users.

Despite the interesting findings, this study has several important limitations. The study has a relatively small sample size from one area of the United States and therefore it is not necessarily representative of all vape shop customers or e-cigarette users. Moreover, the study utilized a convenience sample of vape store customers visiting during a busy but limited specific time period of the day. Although refusal rates were low, the study may suffer from selection bias. In addition, data collection was completed in the spring of 2014. Since that time, the products sold at vape shops have continued to change-with high-powered, variable wattage models becoming more user-friendly. Our data may not reflect the most recent vape shop customer behaviors and preferences. While collecting participants' eCO is a strength of the study, as eCO is known to be a valid measure of smoking status and cessation outcomes comparing favorably with cotinine and other biochemical measures that have longer detection windows ${ }^{19}$, it is less sensitive for non-daily smokers. It is possible that some participants classified as smoking abstinent in this study (i.e., self-reported smoking abstinence and eCO<10ppm) could have been non-daily smokers falsely self-reporting smoking abstinence. However, we believe the motivation for individuals to intentionally misrepresent self-reported data was low, as the survey was anonymous, completed outside of the vape shop, and voluntary. Also, while we received approval to survey customers from the store owner, we did not provide them with the dates we would conduct the survey at their store. Lastly, we did not assess what type of tank-style devices participants were using or their nicotine concentration which may have provided additional insights into vaping behaviors and preferences.

This study provides detailed and important information on the vaping history, behaviors, and preferences of vape shop customers and suggests that further examination of how the unique characteristics of vape shops influence e-cigarette use and smoking cessation is warranted. Furthermore, as the US FDA begins enforcement of new e-cigarette regulations, future research should examine how this not only influences the attitudes and behaviors of the vape shop retailers but also those of the patrons of these establishments.

\section{REFERENCES}

1. Arrazola, R.A., et al., Tobacco use among middle and high school students - United States, 2011-2014. MMWR Morb Mortal Wkly Rep, 2015. 64(14): p. 381-5.

2. King, B.A., et al., Trends in awareness and use of electronic cigarettes among US adults, 2010-2013. Nicotine Tob Res, 2015. 17(2): p. $219-27$. doi: 10.1093/ntr/ntu191.

3. Weaver, S.R., et al., Use of electronic nicotine delivery systems and other tobacco products among USA adults, 2014: results from a national survey. Int J Public Health, 2015. doi: 10.1007/s00038-015-0761-0

4. Herzog B., G.J., Scott A. Tobacco Talk: Vapors/Tanks Driving Next Wave of E-Vapor Growth. Wells Fargo:Wells Frago Securities LLC; 2014.

5. Groskopf, C. What Yelp data reveal about the sudden rise of vape shops in America, 2016. Available from http://qz.com/608469/ what-yelp-data-tells-us-about-vaping/. (accessed March 2016)

6. Sussman, S., et al., Commentary: Forces That Drive the Vape Shop Industry and Implications for the Health Professions. Eval Health Prof, 2015.

7. Lechner, W.V., et al., Effects of Duration of Electronic Cigarette Use. Nicotine Tob Res, 2014. doi: $10.1093 / \mathrm{ntr} / \mathrm{ntu} 061$

8. Tackett, A.P., et al., Biochemically Verified Smoking Cessation and Vaping Beliefs Among Vape Store Customers. Addiction, 2015. doi: 10.1111/add.12878.

9. Lee, Y.O. and A.E. Kim, 'Vape shops' and 'E-Cigarette lounges' open across the USA to promote ENDS.Tob Control. 2015 Jul;24(4):410-2.

doi: 10.1136/tobaccocontrol-2013-051437

10. Lee, Y.O. and A.E. Kim, 'Vape shops' and 'E-Cigarette lounges' open across the USA to promote ENDS. Tob Control, 2015. 24(4): p. 410-2. 


\section{Research Paper}

doi: 10.1136/tobaccocontrol-2013-051437

11. Cheney, M., M. Gowin, and T.F. Wann, Marketing practices of vapor store owners. Am J Public Health, 2015. 105(6): p. e16-21. doi: 10.2105/AJPH.2015.302610.

12. Sussman, S., et al., Consumers' perceptions of vape shops in Southern California: an analysis of online Yelp reviews. Tob Induc Dis, 2014. 12(1): p. 22.

doi: 10.1186/s12971-014-0022-7.

13. Cheney, M.K., M. Gowin, and T.F. Wann, Vapor Store Owner Beliefs and Messages to Customers.Nicotine Tob Res. 2016 May;18(5):694-9. doi: $10.1093 / \mathrm{ntr} / \mathrm{ntv} 129$.

14. Kalkhoran, S. and S.A. Glantz, E-cigarettes and smoking cessation in real-world and clinical settings: a systematic review and metaanalysis. Lancet Respir Med, 2016. 4(2): p. 116-28. doi: 10.1016/S2213-2600(15)00521-4.

15. Sussman, S., et al., Who walks into vape shops in Southern California?: a naturalistic observation of customers. Tob Induc Dis, 2016. 14: p. 18. doi: 10.1186/s12971-016-0082-y

16. Tackett, A.P., et al., Biochemically verified smoking cessation and vaping beliefs among vape store customers. Addiction, 2015. 110(5): p. 868-74. doi: 10.1111 /add.12878.

17. Fagerstrom, K., Determinants of tobacco use and renaming the FTND to the Fagerstrom Test for Cigarette Dependence. Nicotine Tob Res, 2012. 14(1): p. 75-8. doi: $10.1093 / \mathrm{ntr} / \mathrm{ntr} 137$.

18. Transdisciplinary Tobacco Use Research Center Tobacco, D., et al., Time to first cigarette in the morning as an index of ability to quit smoking: implications for nicotine dependence. Nicotine Tob Res, 2007. 9 Suppl 4: p. S555-70.

19. SRNT Subcommittee on Biochemical Verification., Biochemical verification of tobacco use and cessation. Nicotine and Tobacco Research, 2002. 4(2): p. 149-159. doi:10.1080/14622200210123581

20. Sato, S., et al., Optimal cutoff level of breath carbon monoxide for assessing smoking status in patients with asthma and COPD. Chest, 2003. 124(5): p. 1749-54.

21. Pechacek, T.F., et al., The Potential That Electronic Nicotine Delivery Systems Can be a Disruptive Technology: Results From a National Survey. Nicotine Tob Res. 2016 Oct;18(10):1989-97. doi: $10.1093 /$ ntr/ntw102.

22. Nayak, P., C.B. Kemp, and P. Redmon, A Qualitative Study of Vape Shop Operators' Perceptions of Risks and Benefits of E-Cigarette Use and Attitude Toward Their Potential Regulation by the US Food and Drug Administration, Florida, Georgia, South Carolina, or North Carolina, 2015. Prev Chronic Dis, 2016. 13: p. E68. doi: $10.5888 /$ pcd13.160071.

23. Polosa, R., et al., Quit and smoking reduction rates in vape shop consumers: a prospective 12-month survey. Int J Environ Res Public Health, 2015. 12(4): p. 3428-38. doi: 10.3390/ijerph120403428

24. Farsalinos, K.E., et al., Impact of flavour variability on electronic cigarette use experience: an internet survey. Int J Environ Res Public Health, 2013. 10(12): p. 7272-82.

doi: $10.3390 /$ ijerph10127272. 